\title{
A delay differential equation model of mealybugs and green lacewings
}

\author{
Kittipol Jankaew ${ }^{1}$, Chontita Rattanakul ${ }^{1,2^{*}}$ (D) and Warunee Sarika
}

\section{"Correspondence:}

chontita.rat@mahidol.ac.th

'Department of Mathematics,

Faculty of Science, Mahidol

University, Bangkok, Thailand

${ }^{2}$ Centre of Excellence in

Mathematics, Commission on Higher Education, Bangkok,

Thailand

\section{Springer}

\begin{abstract}
In this paper, we propose and analyze a mathematical model of mealybugs and green lacewings with time delay to investigate the population dynamics of mealybugs (a major insect pest of cassava) and green lacewings (a natural enemy of mealybugs) when the time delay in the development of green lacewings is taken in to account. Hopf bifurcation theorem and Routh-Hurwitz criteria are utilized so that the conditions on the model parameters which differentiate various dynamic behaviors of the model are obtained. Computer simulations are also carried out to illustrate our theoretical predictions. Chaotic behavior observed in the field data is also investigated numerically.
\end{abstract}

Keywords: Mathematical model; Mealybugs; Green lacewings; Time delay; Hopf-bifurcation; Periodic solutions

\section{Introduction}

Cassava (Manihot esculenta Crantz) is extensively cultivated as an annual crop in tropical and subtropical regions for its edible starchy storage roots. Apart from being the world's second largest producer of cassava, Thailand is the world largest exporter of cassava products as well [1].

On the other hand, the demand of energy increases continuously. In 2006, it has been reported in [2] that the use of energy worldwide is $11,266.7$ million tons of oil equivalent and increases to 13,276.3 million tons of oil equivalent, which is an increase of $17.84 \%$, and tends to be increasing continuously. However, the resource of energy is limited and it might not be sufficient to reach the demand in the future. Therefore it is necessary to seek for alternative energy. Department of Alternative Energy Development and Efficiency found that dry crops is one of effective choices that can be transmuted to the alternative energy. The most suitable dry crops in Thailand are sugar cane (Saccharum officinarum L.) and cassava (Manihot esculenta L. crantz) that can be transmuted to ethanol. According to [3], 1 ton of cassava roots can be transmuted to 140 liters of ethanol, 1 ton of tapioca chips can be transmuted to 333 liters of ethanol, 1 ton of tapioca starch can be transmuted to 500 liters of ethanol, 1 ton of sugarcane can be transmuted to 70 liters of ethanol, and 1 ton of molasses can be transmuted to 238 liters of ethanol.

In 2008, the outbreak of cassava mealybugs (a major insect pest of cassava) caused severe damage in cassava fields in Thailand and rapidly spread to other areas [4]. According

(c) The Author(s) 2019. This article is distributed under the terms of the Creative Commons Attribution 4.0 International License (http://creativecommons.org/licenses/by/4.0/), which permits unrestricted use, distribution, and reproduction in any medium, provided you give appropriate credit to the original author(s) and the source, provide a link to the Creative Commons license, and indicate if changes were made. 
to Thailand's office of agricultural economics, cassava yields reduced to 23 million tons in the year 2009-2010, which is approximately 10 million tons lower than the expected cassava yields [5]. Mealybug is a major insect pest of cassava. It can destroy up to $54 \%$ of roots and $100 \%$ of leaves in locations of infestation [6]. It damages cassava plants directly by sucking the sap and contaminating the plant with its toxic saliva and indirectly by favoring the development of sooty molds [4]. Without control strategy, mealybugs can reduce cassava yields to less than $80 \%$ [7]. To control the spread of mealybugs, there are many strategies suggested such as insecticide, biological control, or mixed insecticide and biological control. With biological control, the use of mealybug's natural enemies has proved experimentally to be successful [8].

Green lacewing is one of natural enemies mostly used to control the spread of mealybugs. The green lacewing larvae can destroy 100 aphids of mealybugs or more in a week. They damage the prey by using the hollow suckle-shaped jaws that allow them to withdraw fluids from many soft-bodied insects. In this work, the population dynamics of mealybugs and green lacewings will be investigated.

In this paper, we propose and analyze a mathematical model of mealybugs and green lacewings with time delay to investigate the population dynamics of mealybugs (a major insect pest of cassava) and green lacewings (a natural enemy of mealybug) when the time delay in the development of green lacewings is taken in to account.

\section{A mathematical model}

In this paper, we assume that the following nonlinear system of the delay differential equations can be used to investigate the population dynamics of mealybugs and green lacewings:

$$
\begin{aligned}
& \frac{d M}{d t}=a_{1} M\left(1-\frac{M}{k_{1}}\right)-\frac{a_{2} M G}{k_{2}+M}-b_{1} M, \\
& \frac{d G}{d t}=\frac{\alpha_{1} a_{2} M(t-\tau) G(t-\tau)}{k_{2}+M(t-\tau)}-b_{2} G,
\end{aligned}
$$

where $M(t)$ and $G(t)$ account for the population densities of mealybugs and green lacewing larvae at time $t$, respectively; $M(t-\tau)$ and $G(t-\tau)$ account for the population densities of mealybugs and green lacewings at time $t-\tau$, respectively. Here $a_{1}, a_{2}, k_{1}, b_{1}, b_{2}, \alpha_{1}$ are assumed to be positive.

In Eq. (2.1), the term $a_{1} M\left(1-\frac{M}{k_{1}}\right)$ represents the the growth rate of mealybugs, $\frac{a_{2} M G}{k_{2}+M}$ represents the death rate of mealybugs due to the predation by green lacewing larvae, and $b_{1}$ represents the natural death rate of mealybugs.

In Eq. (2.2), since only the green lacewing larvae can prey on mealybugs and it takes approximately 39 days for green lacewing larvae to develop into pupa, an adult that can lay eggs which will develop into green lacewing larvae $[9,10]$, we then assume that the term $\frac{\alpha_{1} a_{2} M(t-\tau) G(t-\tau)}{\left(k_{2}+M(t-\tau)\right)}$ represents the growth rate of green lacewings due to the consumption on mealybugs which incorporates the time delay in the increase of green lacewings; $b_{2}$ represents the natural death rate of green lacewings. 


\section{Modeling analysis}

The points $(0,0)$ and

$$
\left(\frac{b_{2} k_{2}}{\alpha_{1} a_{2}-b_{2}}, \frac{\alpha_{1} k_{2}\left(a_{1} a_{2} \alpha_{1} k_{1}-a_{1} b_{2} k_{1}-a_{1} b_{2} k_{2}-a_{2} b_{1} \alpha_{1} k_{1}+b_{1} b_{2} k_{1}\right)}{k_{1}\left(\alpha_{1} a_{2}-b_{2}\right)^{2}}\right)
$$

are two steady states of the system of Eqs. (2.1)-(2.2).

Firstly, let us consider the local stability of the washout steady state $(0,0)$. The Jacobian matrix of system $(2.1)-(2.2)$ evaluated at the washout steady state $(0,0)$ is

$$
\left.J\right|_{(0,0)}=\left(\begin{array}{cc}
a_{1}-b_{1} & 0 \\
0 & -b_{2}
\end{array}\right) .
$$

We can see that the eigenvalues of $\left.J\right|_{(0,0)}$ are $\lambda_{1}=a_{1}-b_{1}$ and $\lambda_{2}=-b_{2}$, and hence, we obtain the following theorem.

Theorem 1 The washout steady state (0,0) of system (2.1)-(2.2) is locally asymptotically stable if and only if

$$
a_{1}<b_{1} \text { and } b_{2}>0
$$

Proof Since all eigenvalues of $\left.J\right|_{(0,0)}$ will have negative real parts if and only if inequalities in (3.2) hold, the washout steady state $(0,0)$ of system $(2.1)-(2.2)$ is locally asymptotically stable if and only if (3.2) is satisfied.

Next, let us consider the non-washout steady state

$$
\left(\frac{b_{2} k_{2}}{\alpha_{1} a_{2}-b_{2}}, \frac{\alpha_{1} k_{2}\left(a_{1} a_{2} \alpha_{1} k_{1}-a_{1} b_{2} k_{1}-a_{1} b_{2} k_{2}-a_{2} b_{1} \alpha_{1} k_{1}+b_{1} b_{2} k_{1}\right)}{k_{1}\left(\alpha_{1} a_{2}-b_{2}\right)^{2}}\right) \equiv\left(M_{s}, G_{s}\right) .
$$

Note that $M_{s}>0$ and $G_{s}>0$ if and only if

$$
\alpha_{1} a_{2}>b_{2}
$$

and

$$
\left(a_{1} a_{2} \alpha_{1}-a_{1} b_{2}-a_{2} b_{1} \alpha_{1}+b_{1} b_{2}\right)>\frac{a_{1} b_{2} k_{2}}{k_{1}} .
$$

Letting $m=M-M_{s}$ and $g=G-G_{s}$, we will be led to following linearized system of (2.1)-(2.2):

$$
\left(\begin{array}{c}
\dot{m}(t) \\
\dot{g}(t)
\end{array}\right)=J_{s}\left(\begin{array}{c}
m(t) \\
g(t)
\end{array}\right)
$$

where $J_{s}$ is the corresponding Jacobian matrix evaluated at $\left(M_{s}, G_{s}\right)$, namely

$$
J_{s}=\left(\begin{array}{cc}
\frac{\left(a_{1}-b_{1}\right) b_{2}}{\alpha_{1} a_{2}}+\frac{a_{1} b_{2} k_{2}}{\alpha_{1} a_{2} k_{1}}-\frac{2 a_{1} b_{2} k_{2}}{k_{1}\left(\alpha_{1} a_{2}-b_{2}\right)} & -\frac{b_{2}}{\alpha_{1}} \\
\frac{\left(k_{1}\left(a_{1}-b_{1}\right)\left(\alpha_{1} a_{2}-b_{2}\right)-a_{1} b_{2} k_{2}\right) e^{-\lambda \tau}}{a_{2} k_{1}} & -b_{2}\left(1-e^{-\lambda \tau}\right)
\end{array}\right) .
$$


For simplicity, we introduce new parameters by letting

$$
\begin{aligned}
a= & b_{2}-\left(\frac{\left(a_{1}-b_{1}\right) b_{2}}{\alpha_{1} a_{2}}+\frac{a_{1} b_{2} k_{2}}{\alpha_{1} a_{2} k_{1}}-\frac{2 a_{1} b_{2} k_{2}}{k_{1}\left(\alpha_{1} a_{2}-b_{2}\right)}\right), \\
b= & -b_{2}\left(\frac{\left(a_{1}-b_{1}\right) b_{2}}{\alpha_{1} a_{2}}+\frac{a_{1} b_{2} k_{2}}{\alpha_{1} a_{2} k_{1}}-\frac{2 a_{1} b_{2} k_{2}}{k_{1}\left(\alpha_{1} a_{2}-b_{2}\right)}\right), \\
c= & b_{2}\left[\left(\frac{\left(a_{1}-b_{1}\right) b_{2}}{\alpha_{1} a_{2}}+\frac{a_{1} b_{2} k_{2}}{\alpha_{1} a_{2} k_{1}}-\frac{2 a_{1} b_{2} k_{2}}{k_{1}\left(\alpha_{1} a_{2}-b_{2}\right)}\right)\right. \\
& \left.+\frac{\left(k_{1}\left(a_{1}-b_{1}\right)\left(\alpha_{1} a_{2}-b_{2}\right)-a_{1} b_{2} k_{2}\right)}{\alpha_{1} a_{2} k_{1}}\right], \\
d= & -b_{2} .
\end{aligned}
$$

Then, the characteristic equation of $J_{s}$ can be written as

$$
F(\lambda)=\lambda^{2}+a \lambda+b+(c+d \lambda) e^{-\lambda \tau}=0 .
$$

According to the Hopf bifurcation theory, for a periodic solution to exist, it is necessary that Eq. (3.7) has a pair of purely imaginary complex roots $\lambda= \pm i \omega$ for some value of $\tau$. In order that such a pair can be found, one must have $F(i \omega)=0$, that is,

$$
(i \omega)^{2}+a(i \omega)+b+(c+d(i \omega)) e^{(-i \omega) \tau}=0
$$

Equating real and imaginary parts on the left of Eq. (3.8) to zero, we obtain the following equations:

$$
\begin{aligned}
& -\omega^{2}+b+c \cos (\omega \tau)-d \omega \sin (\omega \tau)=0, \\
& a \omega+c \sin (\omega \tau)+d \omega \cos (\omega \tau)=0 .
\end{aligned}
$$

By squaring (3.9) and (3.10), and then adding them, it follows that

$$
\omega^{4}+\left(a^{2}-2 b-d^{2}\right) \omega^{2}+\left(b^{2}-c^{2}\right)=0 .
$$

Let $\eta=\omega^{2}$, then (3.11) can be written as

$$
\delta(\eta) \equiv \eta^{2}+\left(a^{2}-2 b-d^{2}\right) \eta+\left(b^{2}-c^{2}\right)=0 .
$$

Hence, Eq. (3.7) will have a pair of complex solutions $\lambda= \pm i \omega$ provided that (3.12) has a positive real solution $\eta=\omega^{2}>0$.

\section{Lemma 1 If}

$$
b^{2}-c^{2}<0
$$

then Eq. (3.12) has at least one positive real root.

\section{Lemma 2 If}

$$
b^{2}-c^{2} \geq 0
$$


then the necessary conditions that Eq. (3.12) has at least one positive real root are

$$
a^{2}-2 b-d^{2}<0 \text { and }\left(a^{2}-2 b-d^{2}\right)^{2} \geq 4\left(b^{2}-c^{2}\right) .
$$

Without loss of generality, we assume that (3.10) has two positive real roots denoted by $\eta_{1}$ and $\eta_{2}$. Then, (3.9) has two positive roots $\omega_{k}=\sqrt{\eta_{k}}, k=1,2$.

Now, let $\tau_{0}>0$ be the smallest of such $\tau$ for which $\lambda= \pm i \omega$. We substitute $\omega=\omega_{k}$ into Eqs. (3.9)-(3.10) and have

$$
\begin{aligned}
& -\omega_{k}^{2}+b+c \cos \left(\omega_{k} \tau\right)-d \omega_{k} \sin \left(\omega_{k} \tau\right)=0, \\
& a \omega_{k}+c \sin \left(\omega_{k} \tau\right)+d \omega_{k} \cos \left(\omega_{k} \tau\right)=0 .
\end{aligned}
$$

Rewrite Eq. (3.17), we obtain

$$
\sin \left(\omega_{k} \tau\right)=\frac{-a \omega_{k}-d \omega_{k} \cos \left(\omega_{k} \tau\right)}{c} .
$$

Substituting (3.18) into (3.16) and rearranging, we then have

$$
\cos \left(\omega_{k} \tau\right)=\frac{(c-a d) \omega_{k}^{2}-b c}{c^{2}+d^{2} \omega_{k}^{2}} .
$$

Solving for $\tau$, we obtain

$$
\tau_{k}^{(j)}=\frac{1}{\omega_{k}} \arccos \left[\frac{(c-a d) \omega_{k}^{2}-b c}{c^{2}+d^{2} \omega_{k}^{2}}\right]+\frac{(j-1) \pi}{\omega_{k}},
$$

where $k=1,2, j=1,2, \ldots$

Then $\pm i \omega_{k}$ is a pair of purely imaginary roots of Eq. (3.7) with $\tau=\tau_{k}^{(j)}, j=1,2, \ldots$ Thus, we can define

$$
\tau_{0}=\min _{1 \leq k \leq 2, j \geq 1}\left(\tau_{k}^{(j)}\right), \quad \tau_{k}^{(j)}>0
$$

with $\tau_{k}^{(j)}$ defined in Eq. (3.20).

\section{Theorem 2}

(a) If (3.14) holds and

$$
a^{2}-2 b-d^{2} \geq 0 \quad \text { or } \quad\left(a^{2}-2 b-d^{2}\right)^{2}<4\left(b^{2}-c^{2}\right),
$$

then all roots of (3.7) have nonzero real parts for all $\tau \geq 0$.

(b) If

$$
a+d>0 \text { and } b+c>0
$$

and either (3.13) or ((3.14) and (3.15)) holds, then all roots of (3.7) have negative real parts for $\tau \in\left[0, \tau_{0}\right)$ where $\tau_{0}$ is as defined in (3.21). 
Proof (a) Arguing by contradiction, suppose that (3.7) has a root with zero real part for some $\tau \geq 0$. This implies that (3.12) has a positive real root. According to Lemma 2, the necessary conditions that (3.12) has a positive real root are $a^{2}-2 b-d^{2}<0$ and $\left(a^{2}-2 b-\right.$ $\left.d^{2}\right)^{2} \geq 4\left(b^{2}-c^{2}\right)$, which is a contradiction. Hence, all roots of (3.7) have nonzero real parts for all $\tau \geq 0$.

(b) For $\tau=0$, (3.7) is reduced to

$$
F(\lambda)=\lambda^{2}+(a+d) \lambda+(b+c)=0 .
$$

Since all inequalities in (3.23) hold, the Routh-Hurwitz criterion then implies that all roots of (3.7) have negative real parts at $\tau=0$. The continuity of $\lambda(\tau)$ implies that all roots of (3.7) will have negative real parts for values of $\tau$ in some open interval containing $\tau=0$. Hence, there is a $\tau_{c}>0$ such that all roots of (3.7) have negative real parts for $\tau \in\left[0, \tau_{c}\right.$ ).

Since $\tau_{0}$ defined in (3.21) is the minimum of $\tau_{k}^{(j)}$ defined in (3.20) for which the real part of at least one root of (3.7) vanishes provided that (3.22) holds, $\tau_{c}=\tau_{0}$ and the proof is complete.

Theorem 2 implies that if (3.23) and either (3.13) or ((3.14) and (3.15)) hold, the steady state $\left(M_{s}, G_{s}\right)$ of our system of Eqs. (2.1)-(2.2) is stable for some values of $\tau \in\left[0, \tau_{0}\right)$. At $\tau=\tau_{0}, \operatorname{Re}(\lambda(\tau))=0$ by the definition of $\tau_{0}$, and hence the stability of steady state $\left(M_{s}, G_{s}\right)$ is lost at $\tau=\tau_{0}$.

In order for a Hopf bifurcation to occur, and hence a periodic solution of our system of Eqs. (2.1)-(2.2) to be expected, we still need to show that

$$
\left.\frac{d(\operatorname{Re}(\lambda(\tau)))}{d \tau}\right|_{\tau=\tau_{0}} \neq 0
$$

Theorem 3 Suppose that (3.13) or ((3.14) and (3.15)) holds, then $\lambda= \pm i \omega_{0}$ is a pair of purely imaginary roots of (3.7). Moreover,

$$
\left.\frac{d(\operatorname{Re}(\lambda(\tau)))}{d \tau}\right|_{\tau=\tau_{0}} \neq 0
$$

where $\omega_{0}=\left.\omega_{k}\right|_{\tau=\tau_{0}}$, provided that

$$
\delta^{\prime}\left(\beta_{0}\right) \neq 0,
$$

where $\beta_{0}=\omega_{0}^{2}$ and $\omega_{0}=\left.\omega_{k}\right|_{\tau=\tau_{0}}$.

Proof In order to prove that $\left.\frac{d(\operatorname{Re}(\lambda(\tau)))}{d \tau}\right|_{\tau=\tau_{0}} \neq 0$, let us consider Eq. (3.7), namely

$$
F(\lambda)=\lambda^{2}+a \lambda+b+(c+d \lambda) e^{-\lambda \tau}=0 .
$$

Then,

$$
\frac{d F(\lambda)}{d \tau}=\left(2 \lambda+a-(c+d \lambda) \tau e^{-\lambda \tau}+d e^{-\lambda \tau}\right) \frac{d \lambda}{d \tau}-(c+d \lambda) \lambda e^{-\lambda \tau}=0,
$$


and hence,

$$
\left(\frac{d \lambda}{d \tau}\right)^{-1}=\frac{2 \lambda+a}{(c+d \lambda) \lambda e^{-\lambda \tau}}-\frac{\tau}{\lambda}+\frac{d}{(c+d \lambda) \lambda} .
$$

Since $(c+d \lambda) e^{-\lambda \tau}=-\left(\lambda^{2}+a \lambda+b\right)$, we get

$$
\left(\frac{d \lambda}{d \tau}\right)^{-1}=\frac{2 \lambda+a}{-\lambda^{3}-a \lambda^{2}-b \lambda}-\frac{\tau}{\lambda}+\frac{d}{(c+d \lambda) \lambda}
$$

at $\tau=\tau_{0}, \lambda=i \omega_{0}$, and thus

$$
\left.\left(\frac{d \lambda}{d \tau}\right)^{-1}\right|_{\tau=\tau_{0}, \lambda=i \omega_{0}}=\frac{2 i \omega_{0}+a}{a \omega_{0}^{2}+i\left(\omega_{0}^{3}-b \omega_{0}\right)}+i\left(\frac{\tau}{\omega_{0}}\right)+\frac{d}{-d \omega_{0}^{2}+i c \omega_{0}} .
$$

Therefore,

$$
\left.\operatorname{Re}\left(\frac{d \lambda}{d \tau}\right)^{-1}\right|_{\tau=\tau_{0}, \lambda=i \omega_{0}}=\frac{2 \omega_{0}^{2}+a^{2}-2 b}{\omega_{0}^{4}+\left(a^{2}-2 b\right) \omega_{0}^{2}+b^{2}}-\frac{d^{2}}{d^{2} \omega_{0}^{2}+c^{2}} .
$$

Equation (3.11) implies that

$$
\omega_{0}^{4}+\left(a^{2}-2 b\right) \omega_{0}^{2}+b^{2}=d^{2} \omega_{0}^{2}+c^{2},
$$

and then

$$
\left.\operatorname{Re}\left(\frac{d \lambda}{d \tau}\right)^{-1}\right|_{\tau=\tau_{0}, \lambda=i \omega_{0}}=\frac{2 \omega_{0}^{2}+a^{2}-2 b-d^{2}}{d^{2} \omega_{0}^{2}+c^{2}} .
$$

Equation (3.12) implies that

$$
\left.\operatorname{Re}\left(\frac{d \lambda}{d \tau}\right)^{-1}\right|_{\tau=\tau_{0}, \lambda=i \omega_{0}}=\frac{\delta^{\prime}\left(\omega_{0}^{2}\right)}{d^{2} \omega_{0}^{2}+c^{2}} \neq 0 .
$$

Hence, $\left.\frac{d(\operatorname{Re}(\lambda(\tau)))}{d \tau}\right|_{\tau=\tau_{0}} \neq 0$ and the proof is complete.

Theorem 4 If either (3.13) or ((3.14) and (3.15)) holds, then a Hopf bifurcation occurs in our model Eqs. (2.1)-(2.2) for a positive time delay $\tau=\tau_{0}$ given by (3.20) provided that (3.3), (3.4), (3.23), and (3.25) are satisfied.

Proof Since all necessary conditions of the Hopf bifurcation theorem are satisfied, then Eqs. (2.1)-(2.2) undergo a Hopf bifurcation when $\tau=\tau_{0}$, therefore the proof is complete.

\section{Numerical simulations}

In this section, computer simulations of our model Eqs. (2.1)-(2.2) are presented to illustrate our theoretical predictions. Some parametric values are obtained from [10-12].

In Fig. 1 , the parameters $b_{1}$ and $b_{2}$ are taken from [10-12]. The other parameters are chosen to satisfy conditions in Theorem 1. A computer simulation is run for the system 

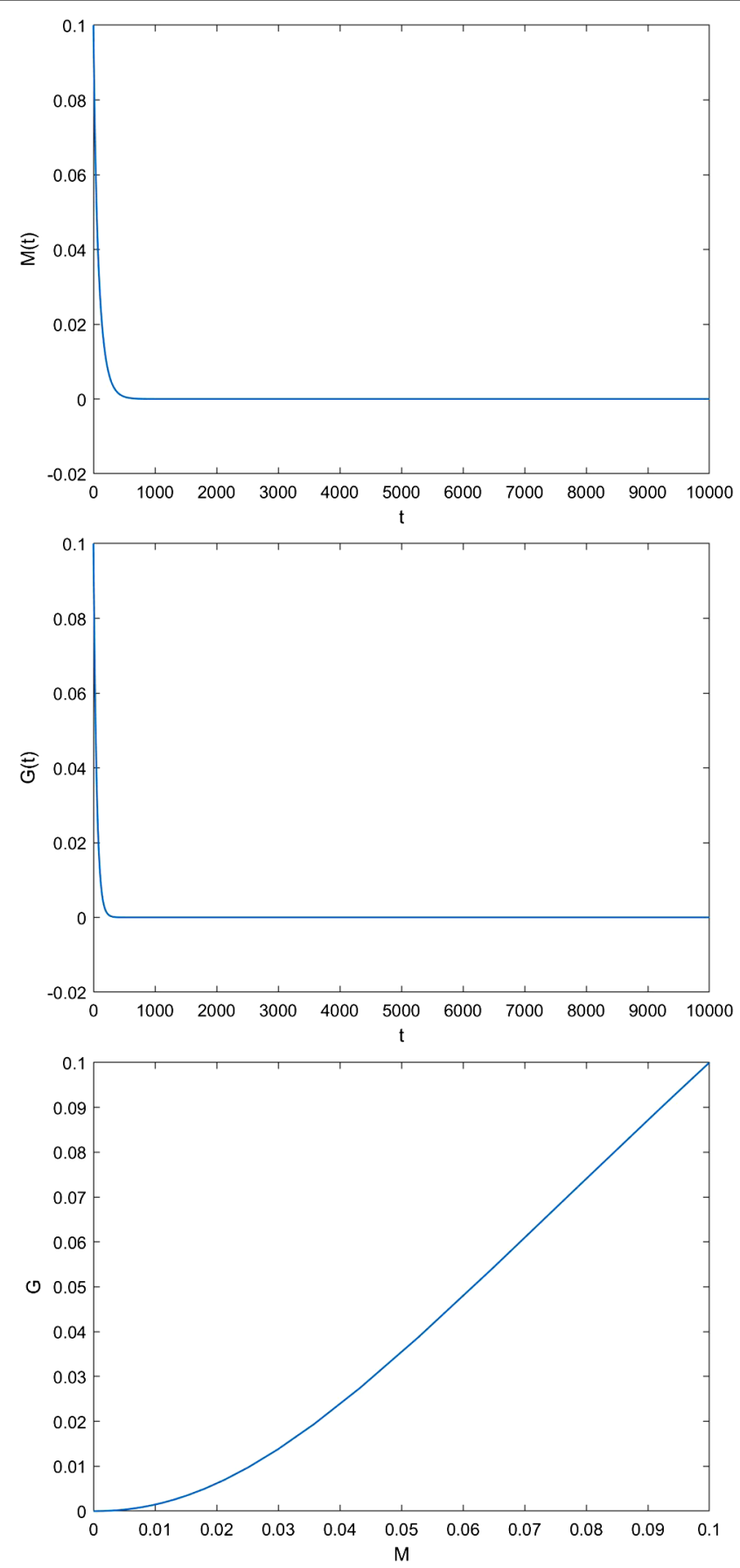

Figure 1 A computer simulation of the system (2.1)-(2.2) with $a_{1}=0.05, a_{2}=0.6937, b_{1}=0.0596$, $b_{2}=0.0206, \alpha_{1}=0.16385, k_{1}=15.64, k_{2}=12.4113, \tau=2$, where $M(0)=0.1$ and $G(0)=0.1$, for which all conditions in Theorem 1 are satisfied. The solution trajectory tends towards a washout steady state as theoretically predicted

(2.1)-(2.2) with $a_{1}=0.05, a_{2}=0.6937, b_{1}=0.0596, b_{2}=0.0206, \alpha_{1}=0.16385, k_{1}=15.64$, $k_{2}=12.4113, \tau=2$, where $M(0)=0.1$ and $G(0)=0.1$, for which all conditions in Theorem 1 are satisfied, showing that the solution trajectory tends towards a washout steady state as theoretically predicted. 

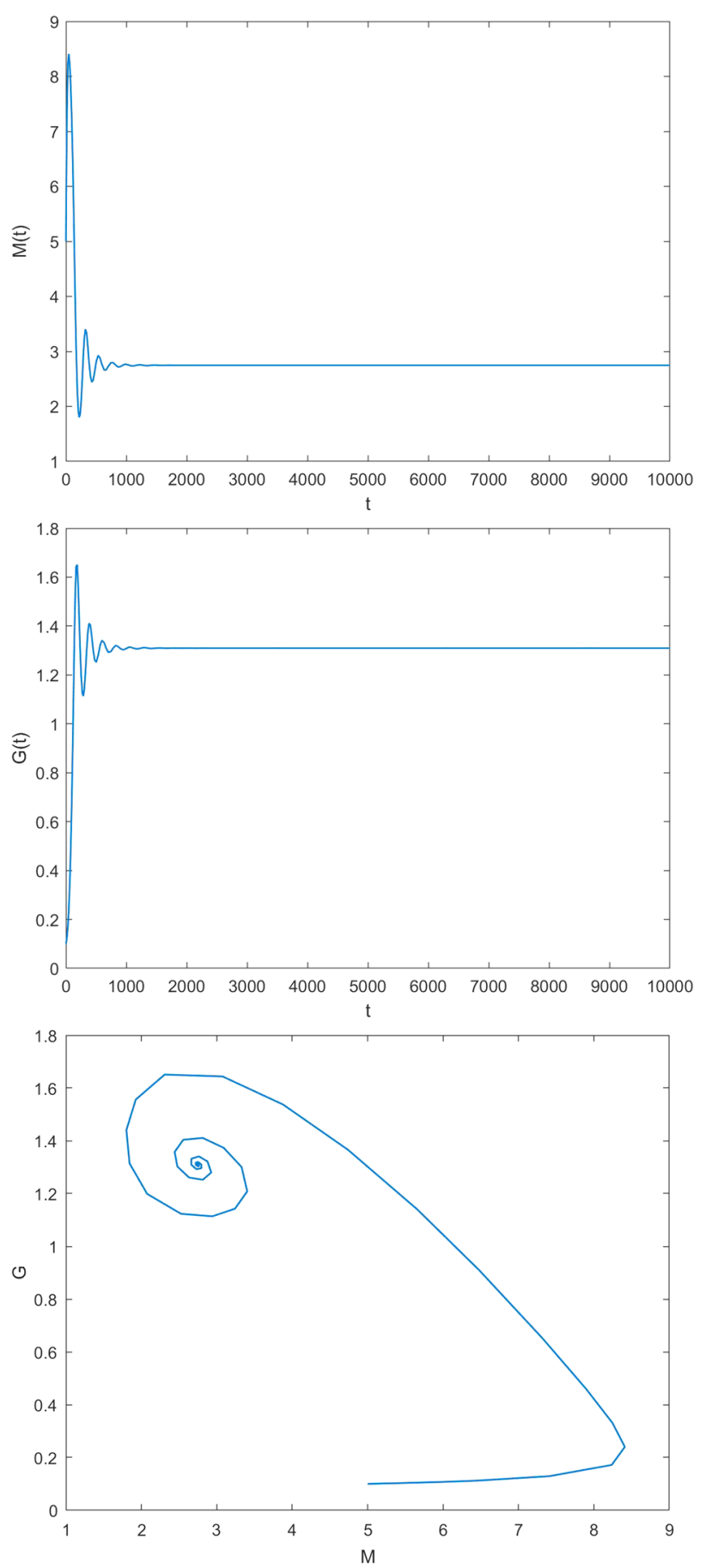

Figure 2 A computer simulation of the system (2.1)-(2.2) with $a_{1}=0.1450, a_{2}=0.6937, b_{1}=0.0596$, $b_{2}=0.0206, \alpha_{1}=0.16385, k_{1}=15.64, k_{2}=12.4113, \tau=3<\tau_{0}=22.5131$, where $M(0)=5$ and $G(0)=0.1$, for which all conditions in Theorem 2(b) are satisfied. The solution trajectory tends towards a non-washout steady state as theoretically predicted

In Figs. 2-8, the parameters $a_{1}, b_{1}$ and $b_{2}$ are taken from [10-12]. The other parameters are chosen to satisfy conditions in Theorems 2 and 4 . 

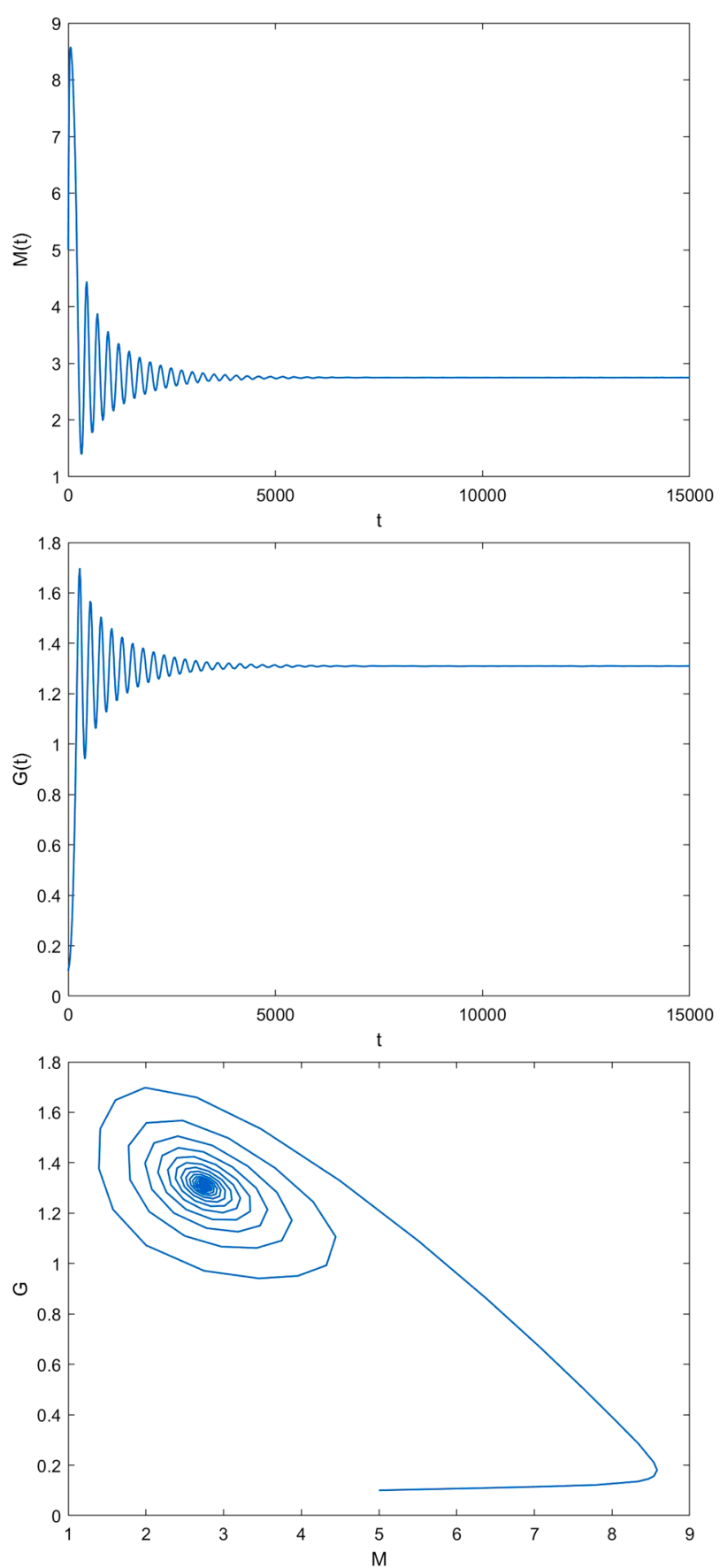

Figure 3 A computer simulation of the system (2.1)-(2.2) with $a_{1}=0.1450, a_{2}=0.6937, b_{1}=0.0596$, $b_{2}=0.0206, \alpha_{1}=0.16385, k_{1}=15.64, k_{2}=12.4113, \tau=22<\tau_{0}=22.5131$, where $M(0)=5$ and $G(0)=0.1$, for which all conditions in Theorem 2(b) are satisfied. The solution trajectory tends towards a non-washout steady state as theoretically predicted

A computer simulation for the system (2.1)-(2.2) with $a_{1}=0.1450, a_{2}=0.6937, b_{1}=$ 0.0596, $b_{2}=0.0206, \alpha_{1}=0.16385, k_{1}=15.64, k_{2}=12.4113, \tau=3<\tau_{0}=22.5131$, where $M(0)=5$ and $G(0)=0.1$, for which all conditions in Theorem $2(\mathrm{~b})$ are satisfied, is shown 

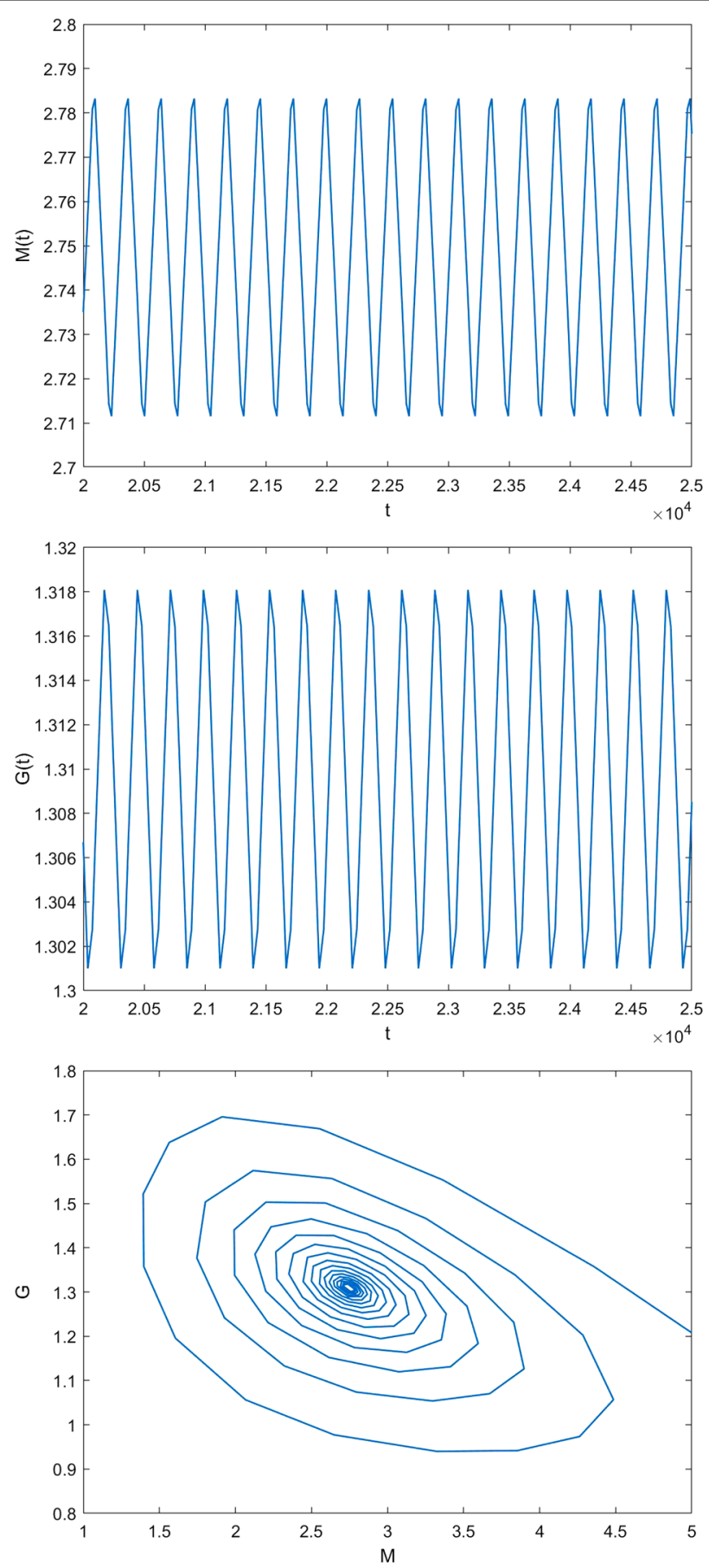

Figure 4 A computer simulation of the system (2.1)-(2.2) with $a_{1}=0.1450, a_{2}=0.6937, b_{1}=0.0596$, $b_{2}=0.0206, \alpha_{1}=0.16385, k_{1}=15.64, k_{2}=12.4113, \tau=\tau_{0}=22.5131$, where $M(0)=5$ and $G(0)=0.1$, for which all conditions in Theorem 4 are satisfied. The solution trajectory exhibits periodic behavior as theoretically predicted

in Fig. 2. The solution trajectory tends towards a non-washout steady state as theoretically predicted.

A computer simulation of the system (2.1)-(2.2) with $a_{1}=0.1450, a_{2}=0.6937, b_{1}=$ $0.0596, b_{2}=0.0206, \alpha_{1}=0.16385, k_{1}=15.64, k_{2}=12.4113, \tau=22<\tau_{0}=22.5131$, where 

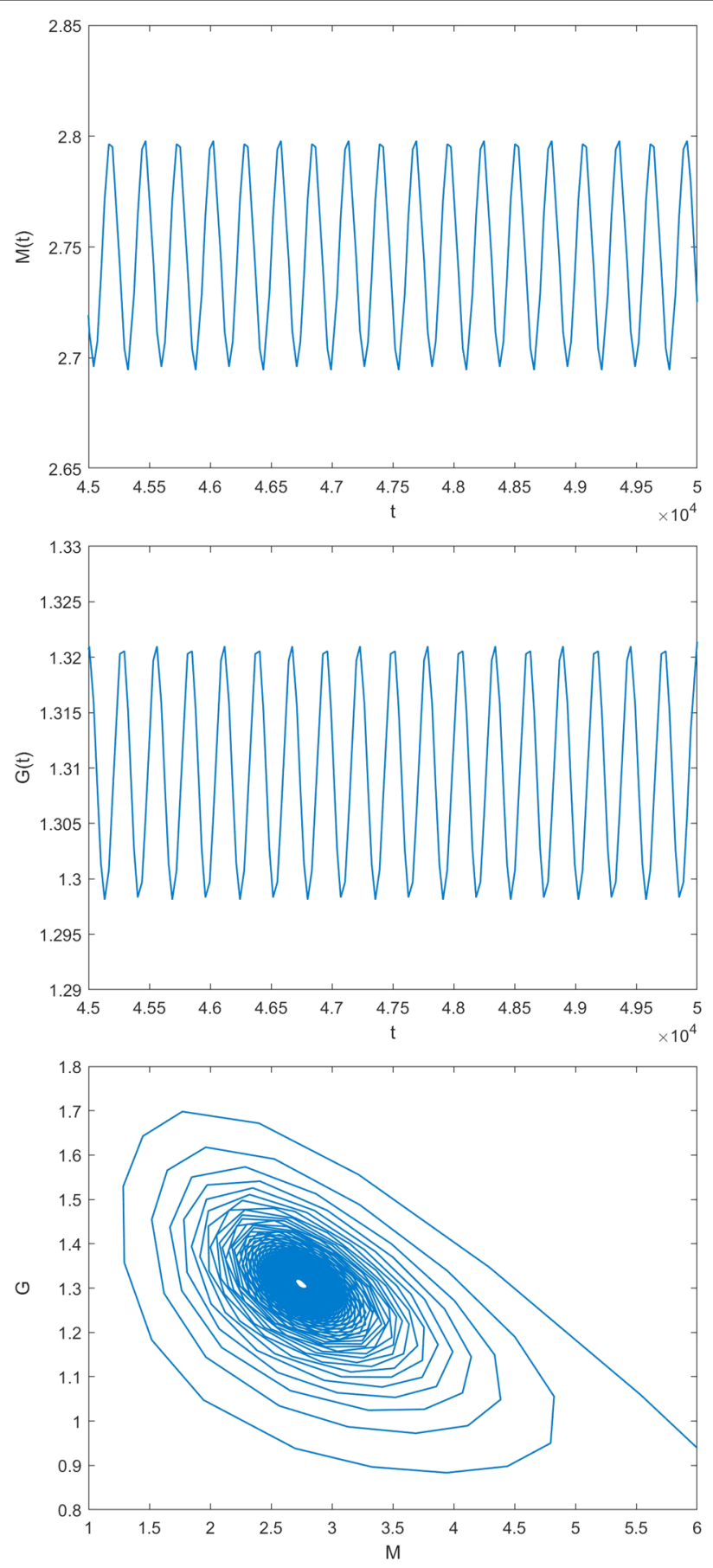

Figure 5 A computer simulation of the system (2.1)-(2.2) with $a_{1}=0.1450, a_{2}=0.6937, b_{1}=0.0596$ $b_{2}=0.0206, \alpha_{1}=0.16385, k_{1}=15.64, k_{2}=12.4113, \tau=30>\tau_{0}=22.5131$, where $M(0)=5$ and $G(0)=0.1$, for which all conditions in Theorem 4 are satisfied. The solution trajectory exhibits periodic behavior as theoretically predicted

$M(0)=5$ and $G(0)=0.1$, for which all conditions in Theorem 2(b) are satisfied, is shown in Fig. 3. The solution trajectory tends towards a non-washout steady state as theoretically predicted. 

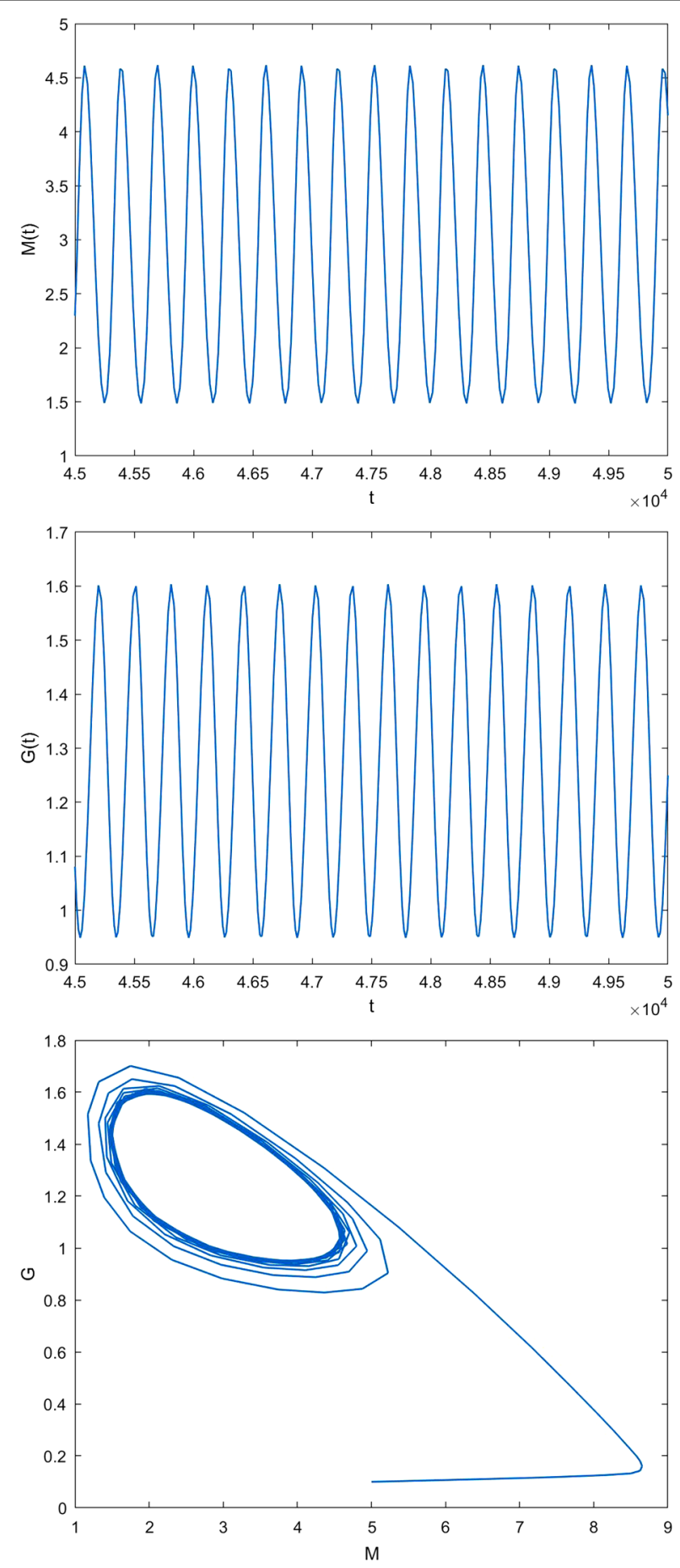

Figure 6 A computer simulation of the system (2.1)-(2.2) with $a_{1}=0.1450, a_{2}=0.6937, b_{1}=0.0596$ $b_{2}=0.0206, \alpha_{1}=0.16385, k_{1}=15.64, k_{2}=12.4113, \tau=35>\tau_{0}=22.5131$, where $M(0)=5$ and $G(0)=0.1$, for which all conditions in Theorem 4 are satisfied. The solution trajectory exhibits periodic behavior as theoretically predicted

A computer simulation of the system (2.1)-(2.2) with $a_{1}=0.1450, a_{2}=0.6937, b_{1}=$ $0.0596, b_{2}=0.0206, \alpha_{1}=0.16385, k_{1}=15.64, k_{2}=12.4113, \tau=\tau_{0}=22.5131$, where $M(0)=$ 

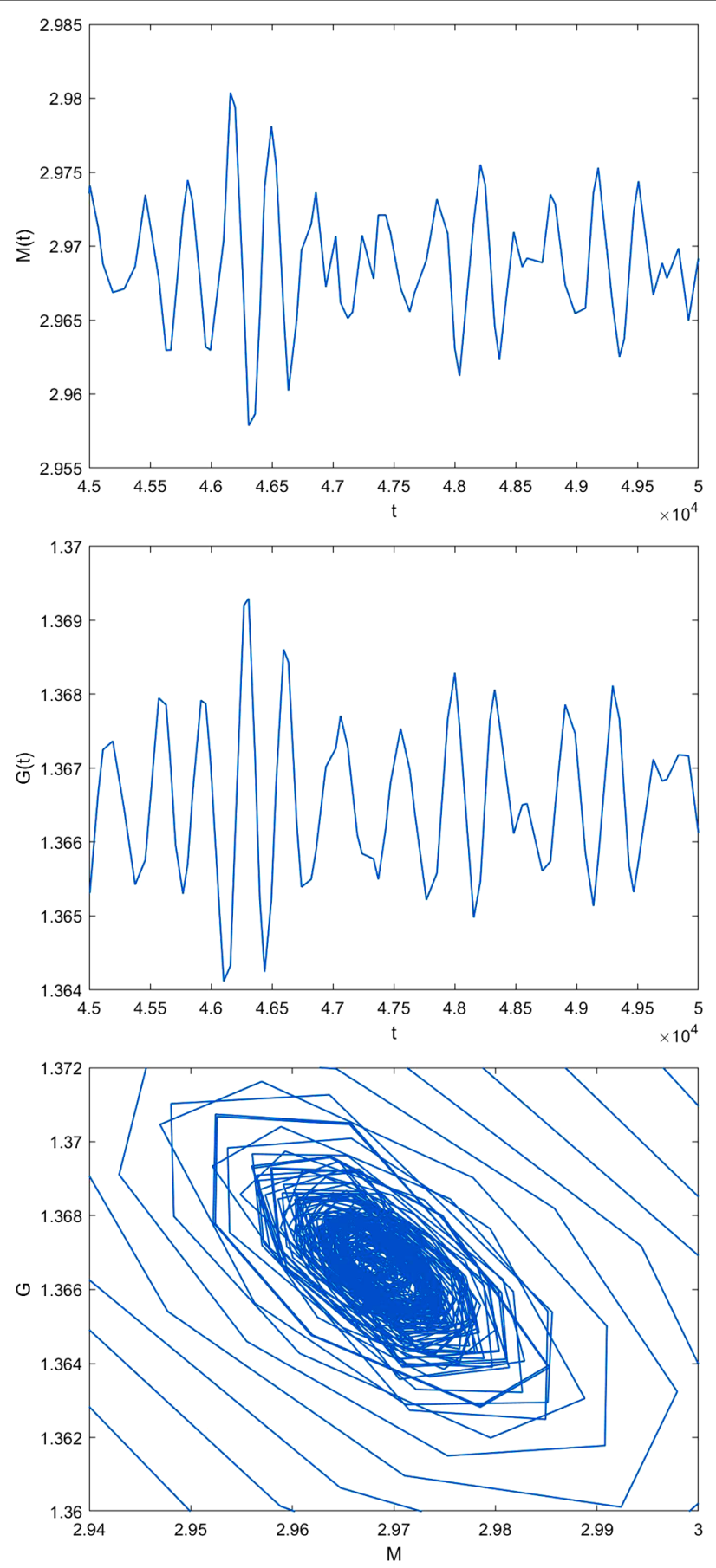

Figure 7 A computer simulation of the system (2.1)-(2.2) with $a_{1}=0.1450, a_{2}=0.6937, b_{1}=0.0596$, $b_{2}=0.0206, \alpha_{1}=0.16385, k_{1}=15.64, k_{2}=12.4113, \tau=39$, where $M(0)=5$ and $G(0)=0.1$. The solution trajectory exhibits chaotic behavior

5 and $G(0)=0.1$, for which all conditions in Theorem 4 are satisfied is shown in Fig. 4. The solution trajectory exhibits periodic behavior as theoretically predicted.

A computer simulation of the system (2.1)-(2.2) with $a_{1}=0.1450, a_{2}=0.6937, b_{1}=$ $0.0596, b_{2}=0.0206, \alpha_{1}=0.16385, k_{1}=15.64, k_{2}=12.4113, \tau=30>\tau_{0}=22.5131$, where 


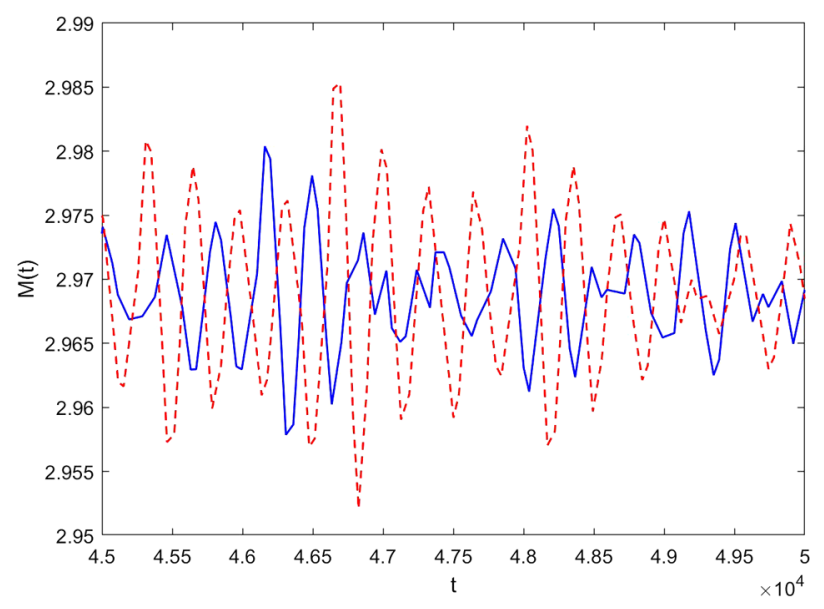

Figure 8 A computer simulation of the system (2.1)-(2.2) with $a_{1}=0.1450, a_{2}=0.6937, b_{1}=0.0596$, $b_{2}=0.0206, \alpha_{1}=0.16385, k_{1}=15.64, k_{2}=13.4113, \tau=39$, where $M(0)=5, M(0)=4.999$, and $G(0)=0.1$. The solution trajectories will stay close only for a short time, before starting to follow noticeably different paths as time passes

$M(0)=5$ and $G(0)=0.1$, for which all conditions in Theorem 4 are satisfied, is shown in Fig. 5 . The solution trajectory exhibits periodic behavior as theoretically predicted.

A computer simulation of the system (2.1)-(2.2) with $a_{1}=0.1450, a_{2}=0.6937, b_{1}=$ $0.0596, b_{2}=0.0206, \alpha_{1}=0.16385, k_{1}=15.64, k_{2}=12.4113, \tau=35>\tau_{0}=22.5131$, where $M(0)=5$ and $G(0)=0.1$, for which all conditions in Theorem 4 are satisfied, is shown in Fig. 6 . The solution trajectory exhibits periodic behavior as theoretically predicted.

A computer simulation of the system (2.1)-(2.2) with $a_{1}=0.1450, a_{2}=0.6937, b_{1}=$ $0.0596, b_{2}=0.0206, \alpha_{1}=0.16385, k_{1}=15.64, k_{2}=12.4113, \tau=39$, where $M(0)=5$ and $G(0)=0.1$, is shown in Fig. 7. The solution trajectory exhibits chaotic behavior.

A computer simulation of the system (2.1)-(2.2) with $a_{1}=0.1450, a_{2}=0.6937, b_{1}=$ $0.0596, b_{2}=0.0206, \alpha_{1}=0.16385, k_{1}=15.64, k_{2}=12.4113, \tau=39$, where $M(0)=5$, $M(0)=4.999$, and $G(0)=0.1$, is as shown in Fig. 8. The solution trajectories will stay close only for a short time, before starting to follow noticeably different paths as time passes.

\section{Conclusion and discussion}

In this paper, a system of nonlinear delay differential equations is utilized to investigate the dynamic behaviors of mealybugs and green lacewings when the time delay on the reproduction of green lacewing larvae is taken into account. The system is then analyzed by using Hopf bifurcation theorem. The conditions on the system parameters that ensure the existence of a periodic solution are obtained. We found that the non-washout steady state of our system is asymptotically stable when the time delay is less than a critical value, i.e., $\tau<\tau_{0}$. A Hopf bifurcation occurs at the critical value of $\tau$, i.e., $\tau=\tau_{0}$. The time delay on the reproduction of green lacewing larvae plays an important role in controlling the population of mealybugs. We can see that when the time delay is less than the critical value, the reproduction of green lacewing larvae is fast enough to control the population of mealybugs to some certain levels. When the time delay is more than the critical value, the population of mealybugs could be controlled to lie within some certain ranges and not at any specific level as we could expect to occur. In addition, our model can also exhibit a chaotic behavior which has been observed in the field data [13]. Hence, our model might 
be modified further to investigate the effects of biological control, insecticide or pathogen in controlling the outbreak of mealybugs in cassava fields.

\section{Funding}

We acknowledge the support by the Centre of Excellence in Mathematics, the Commission on Higher Education, Thailand.

\section{Competing interests}

The authors declare that they have no competing interests.

\section{Authors' contributions}

The first and second author developed and analyzed the model both theoretically and numerically. The third author investigated the literature reviews. All authors read and approved the manuscript.

\section{Publisher's Note}

Springer Nature remains neutral with regard to jurisdictional claims in published maps and institutional affiliations.

Received: 31 January 2019 Accepted: 2 July 2019 Published online: 12 July 2019

\section{References}

1. Chetchuda, C.: Cassava Industry. Krungsri Research, Thailand (2017)

2. Bob, D., Spencer, D.: BP Statistical of World Energy June 2017. London (2017)

3. Cox, J.M., Pearce, M.J., eds.: Wax produced by dermal pores in three species of mealybug (homoptera: Pseudococcidae). Int. J. Insect Morphol. Embryol. 12, 235-248 (1983)

4. Parsa, S., Kondo, T., Winotai, A.: The cassava mealybug (phenacoccus manihoti) in Asia: first records, potential distribution, and an identification key. PLOS ONE 7(10), 1-11 (2012)

5. Mealybugs Cross Thai Border: https://www.phnompenhpost.com/national/mealybugs-cross-thai-border

6. Lema, K.M., Herren, H.R.: The influence of constant temperature on population growth rates of the cassava mealybug, phenacoccus manihoti. Entomol. Exp. Appl. 38(2), 165-169 (1985)

7. Nwanze, K.F.: Relationships between cassava root yields and crop infestations by the mealybug, phenacoccus manihoti. Int. J. Pest Manag. 28, 27-32 (2008)

8. Sarkar, M.A., Suasa-ard, W., Uraichuen, S.: Suitability of different mealybug species (hemiptera: Pseudococcidae) as hosts for the newly identified parasitoid allotropa suasaardi sarkar \& polaszek (hymenoptera: Platygasteridae). Kasetsart J. Nat. Sci. 48, 17-27 (2014)

9. Pappas, M.L., Broufas, G.D., Koveos, D.S.: Effect of prey availability on development and reproduction of the predatory lacewing dichochrysaprasina (neuroptera: Chrysopidae). Ann. Entomol. Soc. Am. 102(3), 437-444 (2009)

10. Pappas, M.L., Koveos, D.S.: Life-history traits of the predatory lacewing dichochrysaprasina (neuroptera: Chrysopidae): temperature-dependent effects when larvae feed on nymphs of myzuspersicae (hemiptera: Aphididae). Ann. Entomol. Soc. Am. 104(1), 43-49 (2011)

11. Barilli, D.R. et al.: Biological characteristics of the cassava mealybug phenacoccus manihoti (hemiptera: Pseudococcidae). Revista Colombiana de Entomología 40(1), 21-24 (2014)

12. Chong, J.H., Roda, A.L., Mannion, C.M.: Life history of the mealybug, maconellicoccushirsutus (hemiptera: Pseudococcidae), at constant temperatures. Environ. Entomol. 37, 323-332 (2008)

13. Wyckhuys, K.A.G. et al.: Continental-scale suppression of an invasive pest by a host-specific parasitoid underlines both environmental and economic benefits of arthropod biological control. PeerJ 6, 5796 (2018)

\section{Submit your manuscript to a SpringerOpen ${ }^{\circ}$ journal and benefit from:}

- Convenient online submission

- Rigorous peer review

- Open access: articles freely available online

- High visibility within the field

Retaining the copyright to your article 